\title{
Effectiveness of antibiotic prophylaxis in patients with centipede stings: a randomized controlled trial
}

\author{
Chanon Changratanakorn', Napasawan Fasawang', \\ Boriboon Chenthanakit ${ }^{1}$, Pakpoom Tansanthong ${ }^{2}$, \\ Chanikarn Mapairoje ${ }^{3}$, Ratree Tunud ${ }^{4}$, Annop Pipopwongpisan ${ }^{4}$, \\ Borwon Wittayachamnankul ${ }^{1}$
}

Department of Emergency Medicine, ${ }^{1}$ Faculty of Medicine, Chiang Mai University, Chiang Mai, ${ }^{2}$ Wiang Nong Long Hospital, Lamphun, ${ }^{3}$ Banthi Hospital, Lamphun, ${ }^{4}$ Li Hospital, Lamphun, Thailand

Objective Centipede stings are a common problem in tropical countries. Current treatment guidelines do not include recommendations for antibiotic prophylaxis to prevent the associated bacterial infection since no previous study has assessed the effectiveness of antibiotic treatment in patients bitten by centipedes. Thus, this study aimed to compare the effectiveness of antibiotic prophylaxis over placebo for the skin infections that occur after a centipede sting.

Methods In this randomized, double-blind, multi-center clinical trial conducted in the emergency departments in four hospitals, patients with any history of a centipede sting were prospectively enrolled and divided randomly into two groups. One group received dicloxacillin and the other a placebo. The primary outcome was the incidence of wound infection 3 to 5 days after the centipede sting.

Results From December 2014 to October 2015, a total of 83 patients were enrolled in the study and were randomized into antibiotic $(n=43)$ and placebo $(n=40)$ groups. Two patients in the antibiotic group developed wound infections, while none showed wound infection in the placebo group (5\% vs. $0 \%$ ). The wound infection rate did not differ significantly between the two groups $(P=0.496)$.

Conclusion Antibiotic prophylaxis may be unnecessary in cases of centipede stings. Proper wound care is an adequate and appropriate treatment for patients with centipede stings. However, the patient should be re-evaluated for detection of secondary bacterial infection.

Keywords Chilopoda; Insect bites and stings; Antibiotic prophylaxis; Wound infection

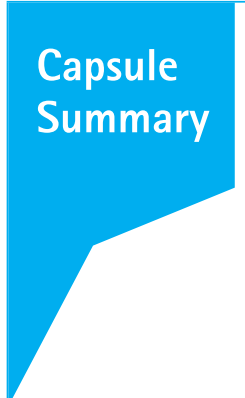

What is already known

Current treatment guidelines do not include a recommendation for antibiotic prophylaxis in centipede stings to prevent an associated bacterial infection since no previous study has been conducted to show the effectiveness of antibiotic prophylaxis in patients with centipede stings.

What is new in the current study

The use of antibiotic prophylaxis in the case of centipede stings may be unnecessary.
elSSN: 2383-4625

Received: 21 August 2020

Revised: 24 October 2020

Accepted: 1 November 2020

Correspondence to:

Borwon Wittayachamnankul

Department of Emergency Medicine,

Faculty of Medicine, Chiang Mai

University, 110 Inthawaroroj, Sriphoom,

Chiang Mai 50200, Thailand

E-mail: borwon.witt@cmu.ac.th

ORCID

https://orcid.org/0000-0003-3465-5534

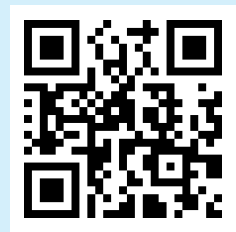

How to cite this article:

Changratanakorn $C_{\text {, Fasawang } N}$ Chenthanakit B, Tansanthong $\mathrm{P}$, Mapairoje C, Tunud R, Pipopwongpisan A, Wittayachamnankul B. Effectiveness of antibiotic prophylaxis in patients with centipede stings: a randomized controlled trial. Clin Exp Emerg Med 2021;8(1):43-47. https://doi.org/10.15441/ceem.20.110

This is an Open Access article distributed under the terms of the Creative Commons Attribution Non-Commercial License (https:// creativecommons.org/licenses/by-nc/4.0/). 


\section{INTRODUCTION}

Centipede stings are a common problem worldwide. 'When attacking humans, a centipede uses its pincer-like maxillipeds, also known as poison claws, to pierce the skin and release the poison. Centipede poison contains histamine and histamine-releaser polypeptides, which are neurotoxins. ${ }^{1}$ The symptoms, which appear 1 to 2 hours after the sting, include pain, swelling, and necrosis. The patient may also experience headache, nausea, and dizziness. In severe, albeit rare, cases, patients may show symptoms of lymphadenitis, cardiac arrhythmia, and acute kidney injury. ${ }^{2}$

In the study region, two species of centipede are commonly found, namely, Scolopendra dehaani and Scolopendra morsitans. ${ }^{3}$ Patients bitten by centipedes experience pain and inflammation of the sting wound. In the absence of recommended medical practices for the treatment of patients with stings, various treatment protocols are applied in clinical practice. The treatment usually consists of both pain relief and antibiotic prophylaxis to prevent infection following the sting. The antibiotics used in wound care should be effective against gram-positive bacteria. ${ }^{4-6} A$ literature review suggested that there are no studies on the use of antibiotic prophylaxis as a preventive strategy against wound infections caused by centipede stings; thus, the role of antibiotic prophylaxis in the treatment of those patients has never been elucidated. The present study aimed to investigate the wound infection rate after centipede stings by comparing the incidence of infection between treatment with antibiotics and placebo.

\section{METHODS}

This study was a prospective, double-blinded, randomized controlled trial conducted between December 2014 and October 2015. We enrolled patients with centipede stings who visited the emergency department of the primary hospital in Maharaj Nakorn Chiang Mai Hospital and three hospitals in Lamphun: Li Hospital, Wiang Nong Long Hospital, and Ban Thi Hospital. The study was approved by the institutional ethical committee of the Faculty of Medicine, Chiang Mai University (EME-2547-02391) and registered in Thai Clinical Trials Registry (TCTR20190613005). All participants gave written informed consent prior to their enrollment.

This study enrolled patients who were 18 years of age or above and were stung by a centipede within 12 hours before visiting the participating emergency departments or hospitals. The main exclusion criteria were allergy to penicillin, a history of antibiotic treatment within 7 days before visiting the hospitals, immunocompromised status (poorly controlled diabetes mellitus, current chemotherapy, leukemia, cancer, human immunodeficiency virus- positive status, humoral immune deficiency, postsplenectomy, usage of systemic steroids), any degree of infection, fever, wounds with blisters, pus or necrosis requiring surgical intervention, and wounds showing a rapid spread of infection or with a clinical description matching necrotizing fasciitis. After explaining the steps involved in the research and clarifying any issues raised, if the patients accepted the terms and agreed to enroll, they signed consent forms for data collection approval. The patients were then divided into two groups by computerized randomization. Thirty data collection forms in separate opaque envelopes were sent to all four participating hospitals. After data collection, the sealed envelopes were returned to the primary hospital for analysis.

Both groups of patients were given standard primary wound care, which involved cleansing with chlorhexidine solution, an injection of a local anesthetic for pain control, and tetanus toxoid prophylaxis as indicated. For this study, dicloxacillin was selected as the antibiotic. ${ }^{4}$ Each group received capsules of the antibiotic or a placebo, the capsules being identical in appearance. The doses were prepared by personnel from the pharmaceutical department, who were not involved in this study in any other role or capacity. The patients were required to take one capsule four times a day after meals for 5 days. They were grouped into those receiving 250-mg capsules of dicloxacillin and those receiving capsules of the placebo. After receiving the capsules, the patients were followed-up daily, and the number of tablets was checked to ensure patient compliance. The patients were also asked about their symptoms and wound status by the general practitioner who met the patients the first time. In addition, the patients were given a phone number to be able to contact the researchers 24 hours a day. Wound infection was ruled out if the wound showed no abnormality or appeared better. However, if the patients experienced any symptoms related to wound infection, including increased pain, increased swelling, increased red/burning sensation, tenderness, or pustules or wounds turning black, follow-up assessments were performed to diagnose the exact status of the wound infection. The presence of wound infection, which was defined as cellulitis, necrosis, or abscess, was diagnosed by the same physician on the basis of presentation of the wound, complete blood count, and bacterial gram staining and bacterial culture of the local wound, which were performed to confirm the presence of infection and the provision of appropriate follow-up treatment. The primary outcome was the incidence of wound infection within 3 to 5 days after the sting.

The sample size was calculated on the basis of the study by Fung et al., ${ }^{5}$ which discussed the follow-up data of patients with centipede stings and reported that $17 \%$ of patients experience wound complications that presumably indicate an infection. Our 
study expected to reduce the rate of infection to 3\% after antibiotic prophylaxis. The sample size calculated as being adequate for the analysis was 45 patients per group.

All normally distributed continuous data were described using the mean values, and the Student t-test was used to analyze the data, with statistical significance indicated by a P-value $<0.05$. Comparisons of nominal data, such as sex, medical illness, infection rate, and complications, between the two groups were performed using a chi-squared test or Fisher exact test.

\section{RESULTS}

From December 2014 to October 2015, a total of 87 patients with centipede stings were admitted to the four emergency departments. Two patients who were randomly selected to receive the antibiotic refused to participate, and nine patients (11\%) were excluded due to follow-up problems. Thus, 74 patients (89\%) were eventually selected for the per-protocol analysis, as shown in Fig. 1.

The baseline data of the two groups, including those related to patient characteristics and medical problems, showed no statistically significant differences, except the data for hypertension. Sig- nificantly fewer patients with hypertension were randomized into the antibiotic group $(P=0.002)$, as shown in Table 1 .

The incidence of wound infection in patients with the centipede stings was two of 40 patients (5\%) in the antibiotic group and zero of 34 patients $(0 \%)$ in the placebo group. The wound infection rate did not differ significantly between the groups $(P=0.496)$,

Table 1. Baseline characteristics of each participant group

\begin{tabular}{lccc}
\hline Characteristics & Antibiotic $(n=40)$ & Placebo $(n=34)$ & P-value \\
\hline Age (yr) & $43.98 \pm 13.19$ & $46.76 \pm 15.07$ & 0.399 \\
No. of days taking medication & $4.60 \pm 1.13$ & $4.56 \pm 1.31$ & 0.885 \\
Sex, male & $22(55)$ & $16(47)$ & 0.496 \\
Medical illness & & & \\
Hypertension & $2(5)$ & $12(35)$ & $0.002^{b)}$ \\
Hypercholesterolemia & $1(3)$ & $1(3)$ & $1.000^{b)}$ \\
Gout & $1(3)$ & $0(0)$ & $1.000^{b)}$ \\
Chronic heart failure & $0(0)$ & $1(3)$ & $0.459^{b)}$ \\
Thyrotoxicosis & $1(3)$ & $0(0)$ & $1.000^{b)}$ \\
Asthma & $1(3)$ & $0(0)$ & $1.000^{b)}$ \\
\hline
\end{tabular}

Values are presented as mean \pm stadard deviation or number (\%).

a) Some patients with more than one medical illness and some with none. ${ }^{b} \mathrm{Cal}-$ culated by Fisher exact test.

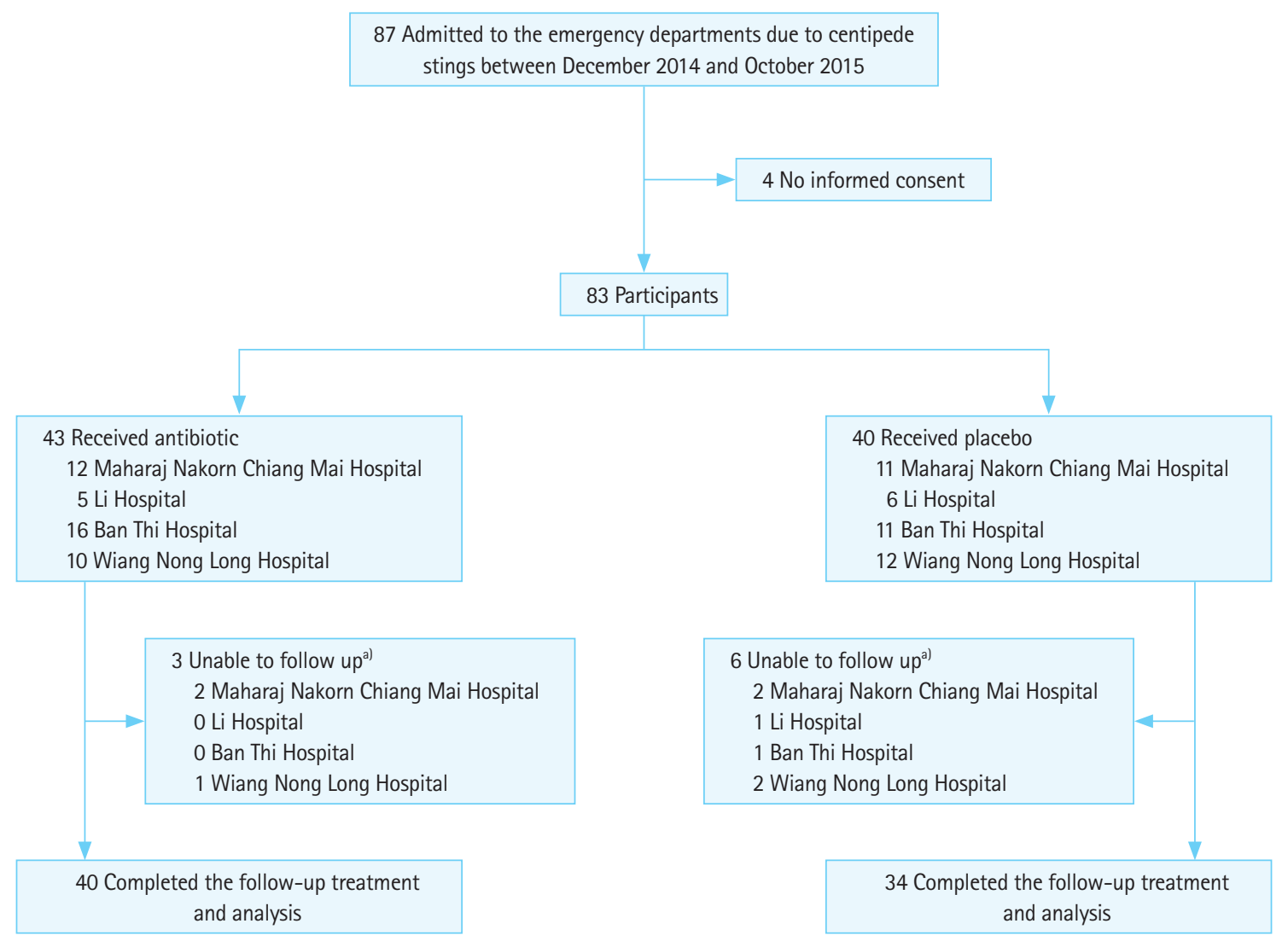

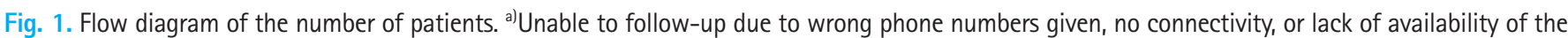
contact given. 
Table 2. Wound infection and wound reaction in patients with centipede stings

\begin{tabular}{lccc}
\hline & $\begin{array}{c}\text { Antibiotic } \\
(\mathrm{n}=40)\end{array}$ & $\begin{array}{c}\text { Placebo } \\
(\mathrm{n}=34)\end{array}$ & P-value \\
\hline $\begin{array}{l}\text { All wound reactions } \\
\text { Clinically infected wound }\end{array}$ & $14(35)$ & $12(35.2)$ & 1.000 \\
Wound reaction from centipede stings & $2(5.0)$ & $0(0)$ & 0.496 \\
$\quad \begin{array}{l}\text { Pruritus } \\
\text { Swelling }\end{array}$ & $7(17.5)$ & $6(17.6)$ & 1.000 \\
Erythema & $5(12.5)$ & $5(14.7)$ & 1.000 \\
& $0(0)$ & $1(2.9)$ & 0.459 \\
\hline
\end{tabular}

Values are presented as number (\%).

as shown in Table 2. The first patient diagnosed with wound infection had cellulitis and lymphangitis 7 days after a centipede bite. Amoxicillin/clavulanic acid (875/125) was prescribed, and the symptoms improved subsequently. The second patient had cellulitis 2 days after a centipede bite. Ceftriaxone was administered intravenously together with chlorpheniramine for 3 days, followed by dicloxacillin for the next 7 days. Subsequently, the infected wound healed and became normal.

\section{DISCUSSION}

Centipedes are arthropods most commonly found in tropical countries. They have a couple of forcipules that can inject their venom into prey. The venom consists of a mixture of cytotoxins, neurotoxins, peptides, enzymes (phospholipase A2, metalloproteases, proteases, transpeptidase, etc.) that causes typical symptoms, including severe pain, erythema, and edema. ${ }^{7,8}$ However, systemic symptoms can also occur, including headache, vomiting, palpitation, cellulitis, necrotizing fasciitis, rhabdomyolysis, and acute myocardial infarction. ${ }^{9}$ The treatment for centipede stings includes pain reduction with ice-packs, local anesthetics or systemic analgesic as needed, and tetanus prophylaxis. ${ }^{10,11}$ To date, there is no conclusive evidence of the effectiveness of antibiotic prophylaxis.

Our findings show that the use of the antibiotic dicloxacillin for the prevention of wound infection from centipede stings may be unnecessary. A previous observational study found that 59\% of patients with centipede stings were given penicillin to prevent wound infection, and 17\% of them later developed wound reaction. ${ }^{5}$ Wound reaction mainly occurred since the toxins in centipede venom include histamine, which results in erythema and swelling. ${ }^{12}$ However, the characteristics of wound infection or the type of antibiotic prescription were not mentioned in that study. Our study, despite including all types of wound reactions, showed no difference in the infection rate between the antibiotic and placebo groups.
The findings also revealed that two patients, accounting for $5 \%$ of the antibiotic group, had wound infections, and both received antibiotics, whereas no infections were observed in the patients receiving placebo. Infections characterized by extension of pain, swelling, and redness 12 hours after the centipede sting may be attributed to gram-negative bacteria, because this study used dicloxacillin, which specifically affects gram-positive cocci. Subsequently, we tried treating the infected patients with broad-spectrum antibiotics (ceftriaxone and amoxicillin-clavulanic acid), which also affect gram-negative bacilli. The infected patients showed significant clinical improvement within 48 hours. This finding indicates that the proper antibiotic for prophylaxis in patients with a centipede sting should include broad-spectrum antibiotics instead of narrow-spectrum antibiotics. However, the results showed no benefit of antibiotics. This may be because the poison of some centipede species contains the homologous peptide scolopendrin I, which can inhibit both gram-positive and gram-negative bacteria, and fungi. ${ }^{13}$

The primary limitation of this study was the number of enrollments. We were unable to recruit sufficient patients to ensure that the findings had sufficient statistical power, and hence were forced to perform per-protocol analysis. Second, wound infection was clinically diagnosed without other confirmatory tests. Therefore, it may be difficult to distinguish from centipede envenomation. Third, the sites of centipede stings may affect the infection and reaction, but we did not record this aspect in our results. Finally, two types of centipedes are commonly found in our study region: Scolopendra dehaani and Scolopendra morsitans. The toxicities of these two species are different, and we could not identify which type of centipede had stung each patient, so we could not determine the differences between these two types of centipedes.

In summary, antibiotic prophylaxis for centipede stings may not be necessary for immunocompetent patients who receive standard wound care. However, patients should be re-evaluated for detection of secondary bacterial infection.

\section{CONFLICT OF INTEREST}

No potential conflict of interest relevant to this article was reported.

\section{ACKNOWLEDGMENTS}

The Faculty of Medicine Research Fund supported this work. 


\section{REFERENCES}

1. Kimura LF, Prezotto-Neto JP, Tavora BC, et al. Local inflammatory reaction induced by Scolopendra viridicornis centipede venom in mice. Toxicon 2013;76:239-46.

2. Otten EJ. Venomous animal injuries. In: Marx JA, Rosen P, editors. Rosen's emergency medicine: concepts and clinical practice. 8th ed. Philadelphia, PA: Elsevier/Saunders; 2014. p.23946.

3. Siriwut W, Edgecombe GD, Sutcharit C, Panha S. The centipede genus Scolopendra in mainland Southeast Asia: molecular phylogenetics, geometric morphometrics and external morphology as tools for species delimitation. PLoS One 2015;10: e0135355.

4. Uzel AP, Steinmann G, Bertino R, Korsaga A. Necrotizing fasciitis and cellulitis of the upper limb resulting from centipede bite: two case reports. Chir Main 2009;28:322-5.

5. Fung HT, Lam SK, Wong OF. Centipede bite victims: a review of patients presenting to two emergency departments in Hong Kong. Hong Kong Med J 2011;17:381-5.

6. Serinken M, Erdur B, Sener S, Kabay B, Cevik A. A case of mortal necrotizing fasciitis of the trunk resulting from a centipede (Scolopendra moritans) bite. Internet J Emerg Med 2004 Dec 31. http://ispub.com/JJEM/2/2/12582.
7. Veraldi S, Cuka E, Gaiani F. Scolopendra bites: a report of two cases and review of the literature. Int J Dermatol 2014;53: 869-72.

8. Undheim EA, Fry BG, King GF. Centipede venom: recent discoveries and current state of knowledge. Toxins (Basel) 2015; 7:679-704.

9. Yildiz A, Biceroglu S, Yakut N, Bilir C, Akdemir R, Akilli A. Acute myocardial infarction in a young man caused by centipede sting. Emerg Med J 2006;23:e30.

10. Essler SE, Julakanti M, Juergens AL. Lymphangitis from Scolopendra heros envenomation: the Texas Redheaded Centipede. Wilderness Environ Med 2017;28:51-3.

11. Guerrero AP. Centipede bites in Hawai'i: a brief case report and review of the literature. Hawaii Med J 2007;66:125-7.

12. Gomes A, Datta A, Sarangi B, Kar PK, Lahiri SC. Occurrence of histamine and histamine release by centipede venom. Indian J Med Res 1982;76:888-91.

13. Wenhua R, Shuangquan Z, Daxiang S, Kaiya Z, Guang Y. Induction, purification and characterization of an antibacterial peptide scolopendrin I from the venom of centipede Scolopendra subspinipes mutilans. Indian J Biochem Biophys 2006; 43:88-93. 